This is the peer reviewed version of the following article: Angew. Chem. 2012,124, 2008-2013, which has been published in final form at: https://doi.org/10.1002/ange.201108092

Bromine Azide

DOI: $10.1002 / a n i e .200(($ will be filled in by the editorial staff $))$

\title{
Festkörperstruktur von Bromazid
}

\section{Benjamin Lyhs, Dieter Bläser, Christoph Wölper, Stephan Schulz*, Georg Jansen}

\author{
Prof. Dr. G. Schmid zum 75. Geburtstag gewidmet
}

Kovalente Azide werden seit über 100 Jahren intensiv untersucht. Stickstoffwasserstoffsäure $\mathrm{HN}_{3}$ wurde erstmalig 1890 von Curtius synthetisiert ${ }^{[1]}$ und ihre molekulare Struktur in der Folgezeit mittels IR und Mikrowellenspektroskopie sowie Gasphasenelektronenbeugung untersucht. ${ }^{[2-4]}$ Kürzlich berichteten Klapötke et al. über die Festkörperstruktur von $\mathrm{HN}_{3} . \mathrm{HN}_{3}$ kristallisiert in einer $\mathrm{AB}$ Schichtstruktur, in der die über intermolekulare Wasserstoffbrücken gebildeten, nahezu planaren Schichten parallel zu (001) gepackt sind. ${ }^{[5]}$

Neben $\mathrm{HN}_{3}$ sind die Halogenazide $\mathrm{XN}_{3}(\mathrm{X}=\mathrm{F}, \mathrm{Cl}, \mathrm{Br}, \mathrm{I})$ die einfachsten kovalenten Azide. ${ }^{[6]}$ Sie wurde bereits frühzeitig experimentell und theoretisch untersucht, insbesondere hinsichtlich ihrer Bindungssituation. $\mathrm{IN}_{3}$ ist monomer in einer $\mathrm{CFCl}_{3}$ Lösung und nimmt eine trans-bent Anordnung in der Gasphase ein, ${ }^{[7]}$ während im Festkörper eine polymere Struktur mit fehlgeordneten Azidgruppen und nahezu identischen I-N Bindungslängen (2.264(23), 2.30(3) $\AA$ ) beobachtet wurde.$^{[8]}$ Leider ist $I_{3}$ bis heute das einzige mittels Einkristallröntgenstrukturanalyse charakterisierte Halogenazid, was nicht zuletzt auf die große Sensibilität der Halogenazide gegenüber geringen Druckschwankungen zurückzuführen ist. Vom Bromazid wurde beispielsweise berichtet, dass es sowohl bei Druckschwankungen von $\Delta p \geq 0.05$ Torr als auch beim Übergang von der flüssigen in die feste Phase explodiert. ${ }^{[9]}$ Trotzdem konnte die Gasphasenstruktur von $\mathrm{BrN}_{3}$, welches ebenfalls eine trans-bent Anordnung zeigt, ${ }^{[9]}$ mittels Elektronenbeugung bestimmt werden. Sie stimmt mit der mittels quantenchemischer Rechnungen ermittelten Struktur überein. ${ }^{[10]}$

Wir interessieren uns seit kurzem für kovalente Hauptgruppenelementazide und berichteten über die Strukturen verschiedener Gruppe 15-Azide wie $\mathrm{Sb}\left(\mathrm{N}_{3}\right)_{3}, \mathrm{Pyr}_{2} \mathrm{Bi}\left(\mathrm{N}_{3}\right)_{3},{ }^{[11]} \mathrm{dem}$ Pentaazidoantimonit-Dianion $\left[\mathrm{Sb}\left(\mathrm{N}_{3}\right) 5^{2-}\right]^{[12]}$ sowie Organoantimondiaziden $\mathrm{RSb}\left(\mathrm{N}_{3}\right)_{2} .^{[13]}$ Wir weiteten diese Studien auf Halogenazide aus und berichten hier über die Einkristallstruktur von $\mathrm{BrN}_{3} 1$.

$$
\mathrm{NaN}_{3}+\mathrm{Br}_{2} \frac{(-196)-(+25){ }^{\circ} \mathrm{C}}{-\mathrm{NaBr}} \mathrm{BrN}_{3} 1
$$

[*] B. Lyhs, D. Bläser, Dr. C. Wölper, Prof. S. Schulz*, Prof. G. Jansen

Fakultät für Chemie, Universität Duisburg-Essen

Universitätsstr. 5-7, S07 S03 C30, D-45117 Essen

Fax: (+) 201-183 3830

E-mail: stephan.schulz@uni-due.de

[**] S. Schulz und B. Lyhs danken dem Fonds der Chemischen Industrie (FCl) für finanzielle Unterstützung sowie einem Doktorandenstipendium (B.L.).

"Supporting Informations" für diesen Artikel sind im WWW unter http://www.angewandte.org oder von den Autoren erhältlich.
Schema 1. Synthese von $\mathrm{BrN}_{3}$

$\mathrm{BrN}_{3} 1$ wurde durch Reaktion von $\mathrm{NaN}_{3}$ mit Brom synthetisiert. Das ${ }^{14} \mathrm{~N}$-NMR Spektrum einer Lösung von $\mathbf{1}$ in $\mathrm{CDCl}_{3}$ zeigt die drei für ein kovalentes Azid erwarteten Resonanzen für $\mathrm{N}_{\alpha}(\delta=-324$, $\left.\Delta \mu_{1 / 2}=118 \mathrm{~Hz}\right), \mathrm{N}_{\beta}\left(\delta=-135, \Delta \mu_{1 / 2}=16 \mathrm{~Hz}\right)$ und $\mathrm{N}_{\gamma}(\delta=-170$, $\Delta \mu_{1 / 2}=25 \mathrm{~Hz}$ ). Diese Werte weichen geringfügig von Literaturwerten für eine Lösung von 1 in $\mathrm{CDCl}_{3} \mathrm{ab},{ }^{[7]}$ stimmen aber sehr gut mit denen einer Lösung in $\mathrm{CH}_{2} \mathrm{Cl}_{2}$ überein, ${ }^{[14]}$ auch wenn die Halbwertsbreiten geringfügig schmaler sind. ${ }^{[14]}$ Das ${ }^{14} \mathrm{~N}-\mathrm{NMR}$ Spektrum von reinem 1 zeigt dagegen deutlich breite Signale.

Table 1. ${ }^{14} \mathrm{~N}$ chemische Verschiebungen von $\mathrm{BrN}_{3}$.

\begin{tabular}{lllll}
\hline Sample & $\mathrm{N}_{\alpha}$ & $\mathrm{N}_{\beta}$ & $\mathrm{N}_{\gamma}$ & Ref. \\
\hline $\mathrm{BrN}_{3}{ }^{[a]}$ & $-324, \Delta \mu_{1 / 2}$ & $-135, \Delta \mu_{1 / 2}$ & $-170, \Delta \mu_{1 / 2}$ & this work \\
& $=118 \mathrm{~Hz}$ & $=16 \mathrm{~Hz}$ & $=25 \mathrm{~Hz}$ & \\
$\mathrm{BrN}_{3}{ }^{[b]}$ & $-319, \Delta \mu_{1 / 2}$ & $-134, \Delta \mu_{1 / 2}$ & $-172, \Delta \mu_{1 / 2}$ & this work \\
& $=288 \mathrm{~Hz}$ & $=22 \mathrm{~Hz}$ & $=118 \mathrm{~Hz}$ & \\
$\mathrm{BrN}_{3}{ }^{[a]}$ & $-349, \Delta \mu_{1 / 2}$ & $-122, \Delta \mu_{1 / 2}$ & $-157, \Delta \mu_{1 / 2}$ & 7 \\
& $=475 \mathrm{~Hz}$ & $=30 \mathrm{~Hz}$ & $=90 \mathrm{~Hz}$ & \\
$\mathrm{BrN}_{3}{ }^{[\mathrm{c}]}$ & $-328, \Delta \mu_{1 / 2}$ & $-142, \Delta \mu_{1 / 2}$ & $-178, \Delta \mu_{1 / 2}$ & 14 \\
& $=220 \mathrm{~Hz}$ & $=65 \mathrm{~Hz}$ & $=80 \mathrm{~Hz}$ & \\
\hline
\end{tabular}

[a] $\mathrm{CDCl}_{3}$ [b] pur (ohne Lösungsmittel) [c] $\mathrm{CH}_{2} \mathrm{Cl}_{2}$

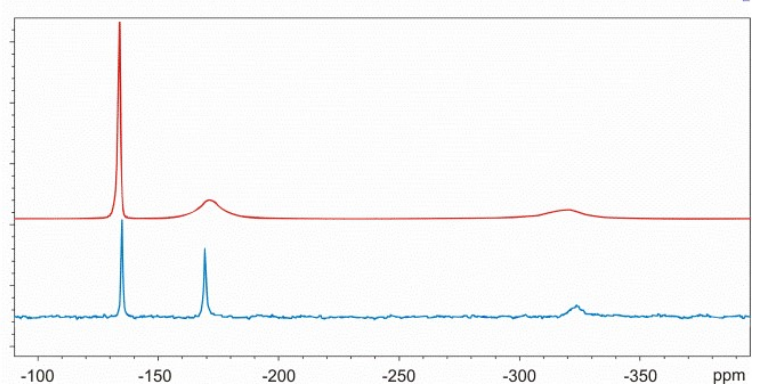

Abbildung 1. ${ }^{14} \mathrm{~N}$ NMR Spektren von $\mathrm{BrN}_{3}$ in $\mathrm{CDCl}_{3}$-Lösung (unten) und ohne zusätzliches Lösungsmittel (oben).

Das Ramanspektrum von flüssigem $\mathrm{BrN}_{3} \mathbf{1}$ zeigt starke Absorptionsbanden der asymmetrischen $\left(v=2146 \mathrm{~cm}^{-1}\right)$ und symmetrischen $\mathrm{N}_{\alpha}-\mathrm{N}_{\beta}-\mathrm{N}_{\gamma}$ Streckschwingungen $\left(v=1274 \mathrm{~cm}^{-1}\right)$ und der $\mathrm{N}_{\alpha}-\mathrm{Br}\left(v=451 \mathrm{~cm}^{-1}\right)$ Streckschwingung. Die Aufnahme des Spektrums wurde durch die ausgeprägte Zersetzungsneigung von 1 unter Bestrahlung erschwert. ${ }^{[15]}$

Einkristalle von 1 wurden direkt auf dem Diffraktometer bei einer Temperatur von $150 \mathrm{~K}$ mittels einer Miniturzonenschmelzanlage mit einem fokussierenden Infrarotlaser gezüchtet. ${ }^{[16]}$ Der IRLaser erlaubt ein kontrolliertes Erwärmen des $\mathrm{BrN}_{3}$, so dass die 
optimalen Wachstumsbedingungen eingestellt werden können. Das erfolgreiche Züchten von Einkristallen von 1 zeigt das große Potential dieser Methode gerade auch für die Strukturbestimmung extrem hitze- und stoßempfindlicher Verbindungen.

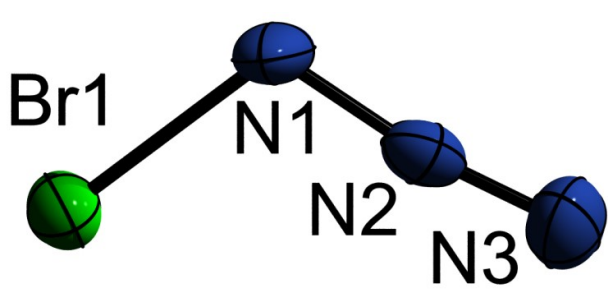

Abbildung 2. Festkörperstruktur von $\mathrm{BrN}_{3}$ 1. Thermalellipsoide mit $50 \%$ Aufenthaltswahrscheinlichkeit.

1 kristallisiert in der tetragonalen Raumgruppe $I 4{ }_{1} \mathrm{Cd}$ mit 16 Molekulen in der Elementarzelle in einer trans-bent Struktur. ${ }^{[17]}$ Der $\mathrm{N}_{\alpha}-\mathrm{N}_{\beta}-\mathrm{N}_{\gamma}$ Bindungswinkel (172.2(11) $\left.{ }^{\circ}\right)$ ist signifikant größer als der Br- $\mathrm{N}_{\alpha}-\mathrm{N}_{\beta}$ Bindungswinkel (108.6(7) ${ }^{\circ}$ ). Die $\mathrm{N}_{\alpha}-\mathrm{Br}$ Bindunglänge (1.916(9) $\AA$ ) ist geringfügig länger als die durch Pyykkö et al. bestimmte Summe der Kovalenzradien $(1.85 \AA),{ }^{[18]}$ stimmt aber sehr gut mit Literaturwerten für Neutralverbindungen mit einer direkten $\mathrm{N}-\mathrm{Br}$ Bindung überein. ${ }^{[19]}$ Die unterschiedlich langen $\mathrm{N}_{\alpha}-\mathrm{N}_{\beta}$ (1.265(9) $\AA$ ) und $\mathrm{N}_{\beta}-\mathrm{N}_{\gamma}$ (1.123(12) $\AA$ ) Bindungslängen belegen eindeutig den kovalenten Bindungscharakter von 1 . Die experimentell ermittelten Bindungslängen und -winkel von 1 stimmen sehr gut mit den mittels Elektronenbeugung bestimmten Werten sowie mit den aus HF-MO and MP2 Rechnungen ermittelten Werten überein. ${ }^{[9]}$ In dieser Arbeit haben wir Rechnungen auf sehr hohem Niveau mittels Coupled-ClusterTheorie mit iterativen Ein- und Zweifach- sowie störungstheoretischen Dreifachanregungen $(\operatorname{CCSD}(T))$ durchgeführt Der Grenzwert eines vollständigen Basissatzes wurde nahezu erreicht mit Hilfe der explizit korrelierten CCSD(T)-F12aMethode, ${ }^{[20]}$ relativistische und Core-Valenzelektronenkorrelationsbeiträge wurden mit dem Douglas-Kroll-Heß-Hamiltonoperator bestimmt. ${ }^{[21,22]}$

Table 2. Ausgewählte strukturelle Parameter von 1.

\begin{tabular}{llll}
\hline & ED $^{[a]}$ & SC $^{[b]}$ & ab initio \\
\hline $\mathrm{N}_{\alpha}-\mathrm{Br}$ & $1.899(6)$ & $1.916(9)$ & 1.894 \\
$\mathrm{~N}_{\alpha}-\mathrm{N}_{\beta}$ & $1.231(22)$ & $1.265(9)$ & 1.250 \\
$\mathrm{~N}_{\beta}-\mathrm{N}_{\gamma}$ & $1.129(22)$ & $1.123(12)$ & 1.134 \\
$\mathrm{~N}_{\alpha}-\mathrm{N}_{\beta}-\mathrm{N}_{\gamma}$ & $170.7(24)$ & $172.2(11)$ & 172.6 \\
$\mathrm{Br}-\mathrm{N}_{\alpha}-\mathrm{N}_{\beta}$ & $109.7(11)$ & $108.6(7)$ & 108.6 \\
\hline
\end{tabular}

[a] Elektronenbeugung [b] Einkristallröntgenstrukturananlyse

Geometrieoptimierungen und Berechnungen harmonischer Frequenzen mit numerischen ersten und zweiten Ableitungen für die Stickstoff- und Brommoleküle demonstrieren die Genauigkeit der verwendeten Methodik: Bindungsabstände und harmonische Frequenzen für ${ }^{14} \mathrm{~N}_{2}$ wurden zu $1.009 \AA$ und $2359 \mathrm{~cm}^{-1}$ erhalten (experimentelle Werte: $1.09768 \AA$ und $2358.57 \mathrm{~cm}^{-1},{ }^{123]}$ ), während für ${ }^{79} \mathrm{Br}_{2} 2.278 \AA$ und $328.5 \mathrm{~cm}^{-1}$ (exp. Werte $2.28105 \AA$ und $325.321 \mathrm{~cm}^{-1}$ ) berechnet wurden. Ohne Berücksichtigung von relativistischen und Core-Valenzkorrelationseffekten wurde $\mathrm{Br}_{2}$ um $0.019 \AA$ länger und seine harmonische Frequenz um $2 \mathrm{~cm}^{-1}$ niedriger gefunden. Die berechneten geometrischen Parameter von $\mathrm{BrN}_{3}$ sind in Tabelle 2 zusammengefasst. Hier ist anzumerken, dass sich die ab-initio-Bindungslängen und -winkel auf die Gleichgewichtsstruktur beziehen, während die experimentellen Daten der Tabelle 2 im Falle der Elektronenbeugungsdaten die Effekte von Schwingungsmittelung bei von Null verschiedener Temperatur sowie im Falle der Einkristallröntgenstruktur zusätzlich die Auswirkungen der Wechselwirkungen mit der Umgebung beinhalten. Dennoch stimmen die Bindungsabstände innerhalb von $0.02 \AA$ und die Bindungswinkel innerhalb von $2^{\circ}$ überein. Die wichtigsten Veränderungen nach Vernachlässigung von relativistischen und Core-Valenzkorrelationseffekten sind eine Verlängerung der N-Br-Bindung um $0.006 \AA$ und eine Aufweitung des Br-N-N-Winkels um 0.3.

Die harmonischen Schwingungsfrequenzen für ${ }^{79} \mathrm{Br}^{14} \mathrm{~N}_{3}$ (187, $475,543,698,1189$ und $\left.2125 \mathrm{~cm}^{-1}\right)$ stimmen innerhalb von $23 \mathrm{~cm}^{-1}$ mit den gemessenen Werten überein, mit Ausnahme der symmetrischen $\mathrm{N}_{\alpha}-\mathrm{N}_{\beta}-\mathrm{N}_{\gamma}$ Streckschwingung, die in den Rechnungen um $85 \mathrm{~cm}^{-1}$ niedriger erhalten wurde. Diese Abweichung kann nicht allein auf relativistische oder Core-Valenzkorrelationseffekte zurückgeführt werden, da diese in unseren Rechnungen die Frequenzen typischerweise um weniger als $5 \mathrm{~cm}^{-1}$ ändern, sondern ist vielmehr nichtharmonischen Korrekturen geschuldet. Die Reaktionsenergie für den Zerfall von $\mathrm{BrN}_{3}$ in $\mathrm{Br}_{2}$ und $\mathrm{N}_{2}(-403.5$ $\mathrm{kJ} / \mathrm{mol}$ bei $0 \mathrm{~K})$ wurde unter Berücksichtigung der Nullpunktsschwingungskorrektur (ZPE) berechnet, die (in harmonischer Näherung) mit $-9.0 \mathrm{~kJ} / \mathrm{mol} \mathrm{zu}$ dem Wert beiträgt, während relativistische und Core-Valenzkorrelationseffekte -0.9 $\mathrm{kJ} / \mathrm{mol}$ beitragen.

Im deutlichen Unterschied zur Festkörperstruktur von $\mathrm{IN}_{3}$, die aus endlosen Ketten aufgebaut ist, die über verbrückende Iodatome mit nahezu identischen I- $\mathrm{N}_{\alpha}$ Bindungslängen (2.264(23), 2.30(3) $\AA$ ) gebildet werden, nimmt $\mathrm{BrN}_{3}$ eine helicale Struktur im Festkörper ein. Ein derartiges Strukturmotiv wurde in der Chemie kovalenter Azide bislang noch nicht beobachtet.

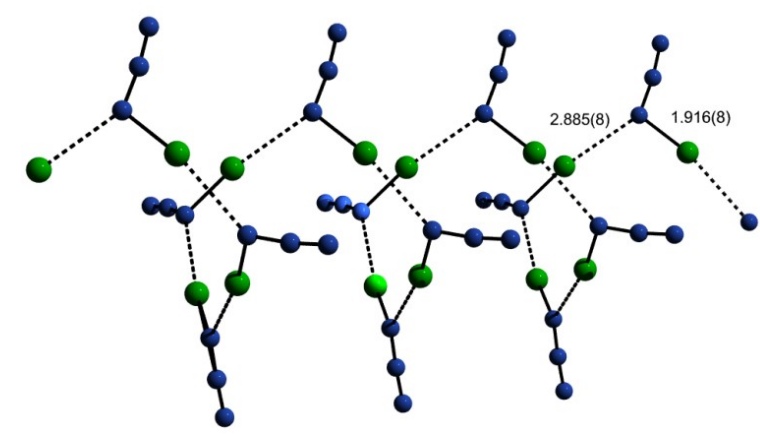

Abbildung 3. Helicale Struktur von 1 aufgrund von intermolekularer Wechselwirkungen zwischen $\mathrm{N}_{\alpha}$ und dem benachbarten Bromatom. Die Helix bildet sich über eine $4{ }_{1}$-Schraubenachse $(y-1 / 2,-x+1, z+1 / 4)$

Die $\quad \mathrm{Br}^{-\mathrm{N}_{\alpha}} \quad(1.916(9) \quad \AA)$ und $\quad \mathrm{Br}^{-\mathrm{N}_{\alpha}}{ }^{\prime} \quad(2.885(8) \quad \AA)$ Bindungslängen differieren um $0.9 \AA$. Der Wert von $2.885(8) \AA$ liegt aber deutlich unterhalb der Summe der van-der-Waals Radien von $\mathrm{Br}$ und $\mathrm{N}(3.38 \AA)^{[20]}$ und weist daher aus kristallographischer Sicht eindeutig auf attraktive Wechselwirkungen hin. Verglichen mit der Festkörperstruktur von $\mathrm{HN}_{3}$ zeigt Bromazid einige frappierende Analogien. $\mathrm{BrN}_{3}$ bildet eine Helix über eine 41Schraubenachse, während für $\mathrm{HN}_{3}$ das gleiche Strukturmotiv mit einer annähernd vierzähligen Symmetrie, jedoch ohne die Translationskomponente, beobachtet wurde. In Folge dessen bildet sich ein achtgliedriger Ring (Abb. 4a in Ref. [5]) aus vier $\mathrm{HN}_{3}$ 
Moleküle anstelle der Helixstruktur von $\mathrm{BrN}_{3}$. Das Gleiche gilt für das Strukturmotiv, das in Abb. 4b in Ref. [5] gezeigt wird. Die Molekülanordnung von $\mathbf{1}$ ist wiederum von einem Ring in eine Helix umgewandelt, allerdings sind die Kontakte in diesem Strukturmotiv bei 1 im Vergleich zu denen bei $\mathrm{HN}_{3}$ weniger eindeutig als attraktive Wechselwirkungen einzustufen. Schwache, aber eindeutig vorhandene, Wasserstoffbrücken verbinden die Moleküle in der $\mathrm{HN}_{3}$ Struktur, begleitet von $\mathrm{N} \cdots \mathrm{N}$ Kontakten gerade unterhalb der Summe der van-der-Waals Radien. ${ }^{[24]}$ In 1 sind an dieser Stelle jedoch nur N $\cdots$ N Kontakte (3.094(16) $\AA$ ) zu finden. Klapötke et al. haben diesen Kontakten schwache attraktive Wechselwirkungen zugeschrieben und dies auf die unterschiedlichen Formalladungen von $\mathrm{N}_{\beta}$ und $\mathrm{N}_{\gamma}$ in einer mesomeren Struktur zurückgeführt, auch wenn diese Grenzstruktur nicht den wichtigsten Beitrag liefert. Zusätzlich beschreiben sie diese Kontakte als Nebeneffekte der Wasserstoffbrücken. Da vergleichbare Kontakte aber nun auch in der Packung von $\mathbf{1}$ auftreten, wo sie nicht auf Wasserstoffbrücken zurückgeführt werden können, erscheint eine schwach-bindende Wechselwirkung möglich. Da sie zudem nicht in $\mathrm{IN}_{3}$ auftreten, erklären sie möglicherweise auch die Entstehung der helicalen Struktur im Festkörper von $\mathrm{BrN}_{3}$, während $\mathrm{IN}_{3}$ eine Kettenstruktur zeigt. Leider führte der Versuch, ein Ramanspektrum von dem Einkristall von 1 aufzunehmen, um diese Kontakte weiter im Detail zu untersuchen, zu einer sofortigen Detonation infolge der Bestrahlung mit dem Ramanlaser, so dass leider keine weiteren experimentellen Aussagen getroffen werden können.

Das dritte Strukturmotiv in der Packung von $\mathrm{HN}_{3}$ (Abb. $4 \mathrm{c}$ in [5]) findet sich ebenfalls in der Packung von 1 wieder. Vier $\mathrm{BrN}_{3}$ Moleküle bilden einen Ring mit zweizähliger Symmetrie. Analog zu den Befunden für $\mathrm{HN}_{3}$ sind die $\mathrm{BrN}_{3}$ Moleküle über zwei schwache und zwei starke Kontakte miteinander verknüpft. Unabhängig davon, ob diese $\mathrm{N} \cdots \mathrm{N}$ Kontakte auf attraktive Wechselwirkungen hindeuten, in Kombination mit den $\mathrm{Br} \cdots \mathrm{N}$ Wechselwirkungen führen sie zur Ausbildung eines dreidimensionalen Netzwerkes (Abb. 4). Zwei dieser, über eine c-Gleitspiegelebene verwandten, Netzwerke sind ineinander verschlungen (Abb. 5).

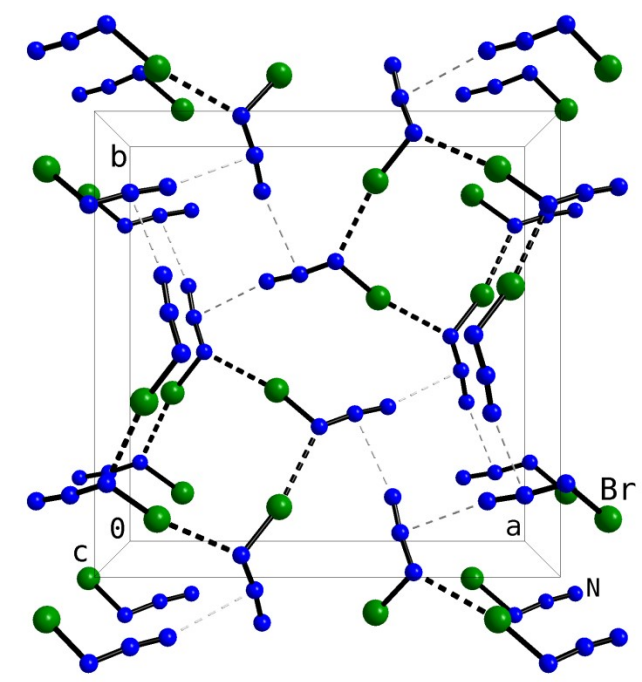

Abbildung 4. Packungbild von 1. Die $\mathrm{Br} \cdots \mathrm{N}$ Wechselwirkungen werden durch dicke gestrichelte Linien illustriert, während die $\mathrm{N} \cdots \mathrm{N}$ Kontakte durch dünnen gestrichelte Linien angedeutet werden. Die aus den $\mathrm{Br} \cdots \mathrm{N}$ Wechselwirkungen resultierenden Helices sind parallel zur c-Achse primitiv gepackt. Unter der Annahme, dass die $\mathrm{N} \cdots \mathrm{N}$ Kontakte auf bindende Wechselwirkungen hinweisen, verbinden sich diese Helices zu einem dreidimensionalen Netzwerk. Die $\mathrm{N} \cdots \mathrm{N}$ Kontakte bilden ihrerseits eine Helix mit $4_{1}$ Symmetrie.
Entlang der zweizähligen Achse parallel zu c erkennt man eine Reihe gestapelter Ringe, z.B. in der Mitte der $a b$-Schicht (andere sind nur unvollständig abgebildet).
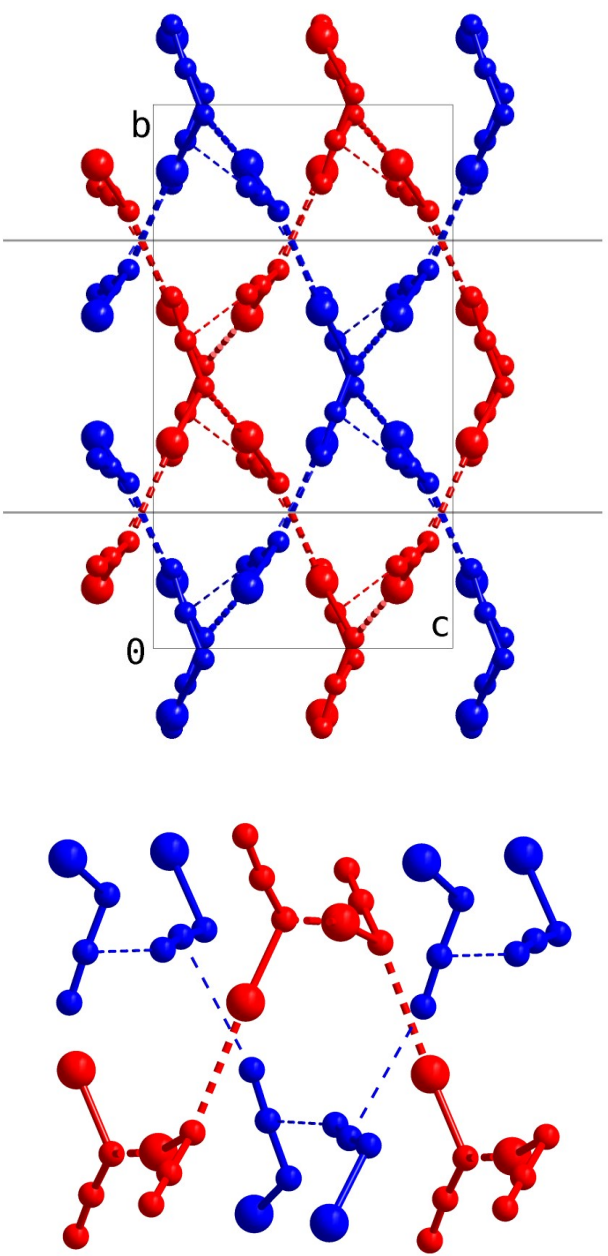

Abbildung 5. Oben: Verschlungene Netzwerke in der Packung von 1. Ein Netzwerk ist in blau, das andere in rot abgebildet. Die hellgrauen Linien markieren die Position der $4{ }_{1}$ Schraubenachse in der Mitte der Helices. Die Netzwerke sind über eine c-Gleitspiegelebene miteinander verwandt. Unten: Details der ineinander verschlungenen Netzwerke. Die durch $\mathrm{Br} \cdots \mathrm{N}$ Wechselwirkungen gebildete Helix (rot) wird von der durch $\mathrm{N} \cdots \mathrm{N}$ Kontakte gebildeten Helix (blau) durchdrungen.

Um die stabilisierende oder destabilisierende Rolle der $\mathrm{N} \cdots \mathrm{N}$ und $\mathrm{Br} \cdots \mathrm{N}$ Kontakte $\mathrm{zu}$ quantifizieren, wurde die Wechselwirkungsenergie eines $\mathrm{BrN}_{3}$ Moleküls mit seinen nächsten Nachbarn im Kristall über CCSD(T)-F12a-Rechnungen mittels derselben ab-initio-Technologie bestimmt, wie sie weiter oben für das Monomer verwendet wurde. ${ }^{[25]}$ Die Wechselwirkungsenergie eines Dimers mit $\mathrm{Br} \cdots \mathrm{N}$-Kontakt in exakt der Geometrie, die auch im Kristall vorliegt, wurde $\mathrm{zu}-13.1 \mathrm{~kJ} / \mathrm{mol}$ berechnet, während für ein Dimer mit N $\cdots \mathrm{N}$ Kontakt $-6.1 \mathrm{~kJ} / \mathrm{mol}$ gefunden wurden. Dieser Befund zeigt, dass letzterer den Kristall in der Tat stabilisiert. Relativistische und Core-Valenzkorrelationseffekte tragen -0.8 $\mathrm{kJ} / \mathrm{mol}$ zum ersteren Wert bei, während ihr Einfluss für den $\mathrm{N} \cdots \mathrm{N}$ Kontakt völlig vernachlässigbar ist. Man sollte beachten, dass die intermolekulare $\mathrm{Br} \cdots \mathrm{N}$-Wechselwirkung ziemlich bedeutsam ist und ca. $60 \%$ der für die Wasserstoffbrückenbindung im dimeren Wassermolekül beobachteten $21 \mathrm{~kJ} / \mathrm{mol}$ beträgt. ${ }^{[2]}$ Diese starken 
Wechselwirkungen sind hauptursächlich verantwortlich für die Stabilität des Kristalls.

Um die Gründe für die Stärke dieser Wechselwirkungen zu beleuchten wurden DFT-SAPT-Berechnungen vorgenommen, $d . h$. Rechnungen mit symmetrieadaptierter intermolekularer Störungstheorie basierend auf einer dichtefunktionaltheoretischen Beschreibung der Monomere. ${ }^{[27]}$ Dabei wird die Wechselwirkungsenergie als Summe von elektrostatischen, Induktions- und Dispersionsenergiebeiträgen erhalten, $\mathrm{zu}$ denen noch repulsive Austauschkorrekturen hinzutreten, die die energetischen Konsequenzen des Antisymmetrieprinzips berücksichtigen. ${ }^{[28]}$ Man sollte beachten, dass in DFT-SAPT keinerlei Multipolentwicklung zur Berechnung dieser Beiträge verwendet wird, sondern sie vielmehr aus Elektronendichten, Dichtematrizen und entsprechenden statischen und dynamischen Responseeigenschaften bestimmt werden. Unter Vernachlässigung relativistischer Effekte wird die Wechselwirkungsenergie der Dimerstruktur mit $\mathrm{Br}^{\cdots} \mathrm{N}_{\alpha-}$ Kontakt $\mathrm{zu}-12.6 \mathrm{~kJ} / \mathrm{mol}$ berechnet, in guter Übereinstimmung mit dem nichtrelativistischen $\operatorname{CCSD}(\mathrm{T})$-Wert von $-12.3 \mathrm{~kJ} / \mathrm{mol}$. Während die Partialladungen einer natürlichen Populationsanalyse $\left(\mathrm{NPA}^{[29]}\right)$ mit +0.19 e für das Br-Atom und mit -0.39 e für das $\mathrm{N}_{\alpha^{-}}$ Atom suggerieren, dass der elektrostatische Beitrag der Hauptbeitrag sein könnte, zeigt Abbildung 6, dass der Dispersionsbeitrag noch etwas bedeutsamer ist. Dies spiegelt die große Polarisierbarkeit des Bromatoms wieder, die auch in der Wichtigkeit des Induktionsbeitrags sichtbar wird. Für die Dimerstruktur mit $\mathrm{N}_{\beta} \cdots \mathrm{N}_{\gamma}$-Kontakt beträgt die DFT-SAPTGesamtwechselwirkungsenergie $\quad-6.0 \mathrm{~kJ} / \mathrm{mol}$, in exzellenter Übereinstimmung mit dem $\operatorname{CCSD}(\mathrm{T})$-Resultat. Wie aufgrund der NPA-Partialladungen von +0.18 e für $\mathrm{N}_{\beta}$ und $+0.02 \mathrm{e}$ für $\mathrm{N}_{\gamma} \mathrm{zu}$ erwarten war, ${ }^{[30]}$ ist der elektrostatische Wechselwirkungsenergiebeitrag sehr viel geringer als der beim $\mathrm{Br}^{\cdots} \mathrm{N}_{\alpha}$-Kontakt. Aufgrund der unvollständigen Abschirmung der Anziehung zwischen Kernen und Elektronen der verschiedenen Moleküle durch die ElektronElektron- und Kern-Kern-Abstoßungen handelt es sich dennoch um einen anziehenden Beitrag. Nichtsdestotrotz ist die Dispersionsenergie der dominante stabilisierende Beitrag im Falle des $\mathrm{N}_{\beta} \cdots \mathrm{N}_{\gamma}$-Kontaktes.

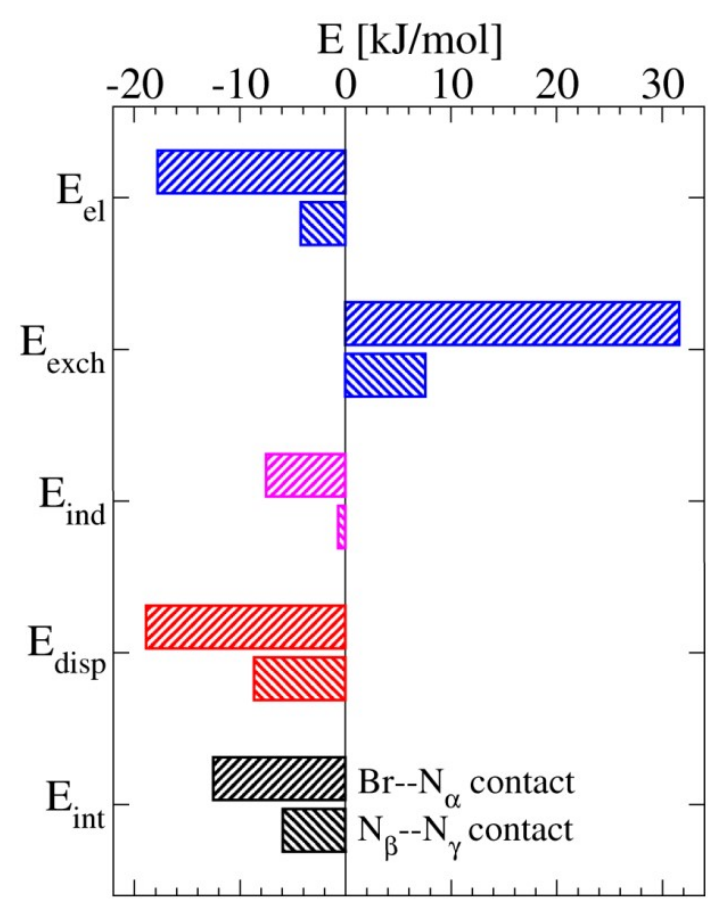

Abbildung 6. Elektrostatische, $\mathrm{E}_{\mathrm{el}}$, Austausch-, $\mathrm{E}_{\mathrm{exch}}$, Induktions-, $\mathrm{E}_{\mathrm{int}}$, und Dispersions-, $E_{\text {disp }}$, DFT-SAPT-Beiträge zur totalen

Wechselwirkungsenergie, $E_{\text {int }}$, für ein Dimer mith $B r \cdots N_{\alpha}$ und $N_{\beta} \cdots N_{\gamma}$ Kontakten in der im Kristall beobachteten Geometrie.

\section{Experimenteller Teil}

Bromazid ist potentiell toxisch und kann unter verschiedenen Bedingungen explosionsartig zerfallen! Es sollte mit größter Sorgfalt und nur in kleinen Mengen $(<2 \mathrm{mmol}$ ) mit entsprechender Schutzausrüstung (Sicherheitsschilde, Schutzbrille, Gesichtsschutz, Lederhandschuhe, Schutzmantel (Leder) und Ohrschützer) gehandhabt werden. Wann immer möglich sollten Teflonbehälter benutzt werden, um im Explosionsfall Splitterbildung vorzubeugen. Das Ignorieren der geschilderten Schutzvorkehrungen kann erhebliche Schäden nach sich ziehen. Alle Reaktionen wurden in FEP-Röhrchen durchgeführt. Flüchtige Verbindungen wurden an einer kombinierten Stahl-Teflon-FEP Vakuumapparatur abgefüllt, nichtflüchtige Substanzen unter Argon in einer Glovebox. $\mathrm{CDCl}_{3}$ wurde sorgfältig über Molekularsieb (3 $\AA$ ) getrocknet und vor Gebrauch entgast. Die ${ }^{14} \mathrm{~N}$ NMR Spektren wurden auf einem Bruker Avance 300 Spektrometer bei $25^{\circ} \mathrm{C}$ mit $21.7 \mathrm{MHz}$ aufgenommen und auf externes $\mathrm{CH}_{3} \mathrm{NO}_{2}\left(\delta\left({ }^{14} \mathrm{~N}\right)=0\right)$ referenziert. Die Ramanspektren wurden mit einem Bruker FT-Ramanspektrometer RFS 100/S mit der $1064 \mathrm{~nm}$ Linie eines Nd:YAG Lasern gemessen. Die zurückgestreute $\left(180^{\circ}\right)$ Strahlung wurde analysiert (Stoke-Bereich: 0 to $3500 \mathrm{~cm}^{-1}$ ). Die flüssige Probe wurde in abgeschmolzenen Glaskapillaren (400 Scans und einer Auflösung von $2 \mathrm{~cm}^{-1}$ ) und einer Laserleistung von $40 \mathrm{~mW}$ vermessen. Leider führte der Versuch, ein Ramanspektrum des $\mathrm{BrN}_{3}$ Einkristalls direkt auf dem Diffraktometer zu messen (80 $\mathrm{mW}$ Leistung), zu einer spontanen Explosion.

$\mathrm{BrN}_{3}$ (1). $0.14 \mathrm{~g}(2.15 \mathrm{mmol}) \mathrm{NaN}_{3}$ wurden in der Glovebox in ein FEP Reaktionsröhrchen abgefült. $80 \mu \mathrm{L}(1.55 \mathrm{mmol}) \mathrm{Br}_{2}$ wurde auf das $\mathrm{NaN}_{3}$ bei $-196{ }^{\circ} \mathrm{C}$ aufkondensiert. Das Röhrchen wurde anschließend langsam auf Raumtemperatur erwärmt und bei dieser Temperatur für 30 Minuten gehalten. Anschließend wurde die resultierende Reaktionsmischung langsam auf $-15{ }^{\circ} \mathrm{C}$ abgekühlt und das $\mathrm{BrN}_{3}$ in ein zweites, auf $-80^{\circ} \mathrm{C}$ gekühltes, FEP Röhrchen umkondensiert. Dieser Vorgang wurde zweimal wiederholt, um reines $\mathrm{BrN}_{3} \mathrm{zu}$ erhalten. 
Raman $\left(40 \mathrm{~mW}, 25^{\circ} \mathrm{C}, 400\right.$ Scans): $v=2146,1273,451,303, \mathrm{~cm}^{-1}$. ${ }^{14} \mathrm{~N}\left\{{ }^{1} \mathrm{H}\right\}\left(\mathrm{CDCl}_{3}\right): \delta=-135\left(\mathrm{~s}, \mathrm{~N}_{\beta}, \Delta v_{1 / 2}=16 \mathrm{~Hz}\right),-170\left(\mathrm{~s}, \mathrm{~N}_{\mathrm{\gamma}}, \Delta \mathrm{v}_{1 / 2}=25\right.$ $\mathrm{Hz}$ ), $-324\left(\mathrm{~s}, \mathrm{~N}_{\alpha}, \Delta v_{1 / 2}=118 \mathrm{~Hz}\right) .{ }^{14} \mathrm{~N}\left\{{ }^{1} \mathrm{H}\right\}$ (pure $\mathrm{BrN}_{3}$ ): $\delta=-134\left(\mathrm{~s}, \mathrm{~N}_{\beta}\right.$, $\left.\Delta v_{1 / 2}=22 \mathrm{~Hz}\right),-172\left(\mathrm{~s}, \mathrm{~N}_{\mathrm{y}}, \Delta v_{1 / 2}=182 \mathrm{~Hz}\right),-319\left(\mathrm{~s}, \mathrm{~N}_{\mathrm{a}}, \Delta v_{1 / 2}=288\right.$ $\mathrm{Hz})$.

Received: ((will be filled in by the editorial staff))

Published online on ((will be filled in by the editorial staff))

Keywords: Kovalente Azide $\cdot \mathrm{XRD} \cdot$ Bromazid $\cdot a b$ initio Rechnungen

[1] N. N. Greenwood, A. Earnshaw, In Chemistry of the Elements, 1st ed., Pergamon Press, Amsterdam, 1984, p. 12.

[2] E. H. Eyster, J. Chem. Phys. 1940, 8, 135-142.

[3] E. Amble, B. P. Daley, J. Chem. Phys. 1950, 18, 1422-142.

[4] V. R. Schomaker, A. Spurr, J. Am. Chem. Soc. 1942, 64, 1184-1187.

[5] J. Evers, M. Göbel, B. Krumm, F. Martin, S. Medvedyev, G.

Oehlinger, F. X. Steemann, I. Troyan, T. M. Klapötke, M. I. Eremets J. Am. Chem. Soc. 2011, 133, 12100-12105.

[6] Für Referenzen sehe: I. C. Tornieporth-Oetting, T. M. Klapötke, Angew. Chem. 1995, 107, 559-568; Angew. Chem. Int Ed. 1995, 34, 511-520.

[7] P. Geissler, T. M. Klapötke, H.-J. Kroth, Spectrochim. Acta 1995, 51A, 1075-1078.

[8] P. Buzek, T. M. Klapötke, P. v. R. Schleyer, I. C. Tornieporth-Oetting, P. S. White, Angew. Chem. 1993, 105, 289-290; Angew. Chem. Int Ed. 1993, $32,275-276$.

[9] M. Hargittai, I. C. Tornieporth-Oetting, T. M. Klapötke, M. Kolonits, I. Hargittai, Angew. Chem. 1993, 105, 773-774; Angew. Chem. Int Ed. 1993, $32,759-760$.

[10] a) M. Otto, S. D. Lotz, G. Frenking, Inorg. Chem. 1992, 31, 3647-

3655; b) A. Schulz, I. C. Tornieporth-Oetting, T. M. Klapötke, Inorg. Chem. 1995, 34, 4343-4346.

[11] S. Schulz, B. Lyhs, G. Jansen, D. Bläser, C. Wölper, Chem. Commun. 2011, 47, 3401-3403.

[12] B. Lyhs, G. Jansen, D. Bläser, C. Wölper, S. Schulz, Chem.-Eur. J. 2011, 17, 11394.

[13] B. Lyhs, D. Bläser, C. Wölper, S. Schulz, Chem. Eur. J. 2011, 17, 4914-4920.

[14] T. M. Klapötke, Polyhedron 1997, 16, 2701-2704.

[15] Details werden im "Supporting Information File" gegeben.

[16] R. Boese, M. Nussbaumer, "In Situ Crystallisation Techniques", In "Organic Crystal Chemistry", Ed. D.W. Jones, Oxford University Press, Oxford, England, (1994) 20-37.

[17] Bruker AXS SMART Diffraktometer mit APEX2 Detektor (MoK $\alpha$ Strahlung, $\lambda=0.71073 \AA ; T=150(1) \mathrm{K}$ ). Die Struktur wurde mittels Direkter Methoden gelöst (SHELXS-97, G. M. Sheldrick, Acta Crystallogr. Sect. $A$ 1990, 46, 467) und gegen $\mathrm{F}^{2}$ verfeinert. Alle Atome wurden anisotrop verfeinert. Eine semiempirische Absorptionskorrektur wurde nach dem Multi-Scan-Verfahren (Bruker AXS APEX2) durchgeführt. Ein geeigneter Einkristall wurde mittels eines in-situ Zonenschmelzverfahrens in einer Quarzkapillare mit einer IR-Laser gezogen. Das experimentelle Setup erlaubt lediglich $\omega$ Scans mit $\chi$ von $0^{\circ}$. Jede andere Orientierung hätte die Kapillare aus dem Kühlbereich entfernt und zu einem Schmelzen des Kristalls geführt. Diese experimentelle Anordnung limitiert die Vollständigkeit der Daten je nach Kristallsystem auf $65 \%$ bis $90 \%$. 1: $\mathrm{BrN}_{3}, \mathrm{M}=121.94$, gelber Kristall $(0.27 \times 0.05 \times 0.03 \mathrm{~mm})$; tetragonal, Raumgruppe $I 4_{1} c d ; a=13.1873(12), b$ $=13.1873(12), c=7.266(3) \AA ; \alpha=\beta=\gamma=90^{\circ}, V=1263.6(5) \AA^{3} ; Z=16 ; \mu$ $=12.736 \mathrm{~mm}^{-1} ; \rho_{\text {ber. }}=2.564 \mathrm{~g} \mathrm{~cm}^{-3} ; 3541$ Reflexe $\left(2 \theta_{\max }=54^{\circ}\right), 586$ unabhängig $\left(R_{\text {int }}=0.1904\right) ; 37$ Parameter, 1 Restraint; Größtes Max./Min. in der finalen Differenz-Fourier-Synthese $0.620 \mathrm{e}^{-3} /-0.613 \mathrm{e} \AA^{-3} ; \mathrm{max} . / \mathrm{min}$. Transmission $0.75 / 0.17 ; R_{1}=0.0423(\mathrm{I}>2 \sigma(\mathrm{I})), w R_{2}($ all data $)=0.0616$. Flack absoluter Strukturparameter 0.11(6) in der finalen

Strukturfaktorberechnung (a) H. D. Flack, Acta Crystallogr. Sect. A 1983, 39 , 876-881. b) G. Bernadinelli, H. D. Flack, Acta Crystallogr. Sect. A 1985, 41, 500-511.).

Details der Kristallstrukturbestimmung von 1 können beim

Fachinformationszentrum Karlsruhe, 76344 Eggenstein-Leopoldshafen,
Deutschland (Fax: +49-7247-808-666; E-mail: crysdata@fiz-karlsruhe.de,) unter Angabe der Nummer CSD-423741 angefordert werden.

[18] P. Pyykkö, M. Atsumi, Chem.-Eur. J. 2009, 15, 186-197.

[19] Eine Suche in der Cambridge Structure Database (CSD) search (Version 5.32 Update August 2011) mit ConQuest (Version 1.13) ergab 41 Verbindungen (30 neutral, 11 ionisch) mit einer N-Br Bindung und einer durchschnittlichen Bindungslänge von $1.89 \AA$.

[20] a) T. B. Adler, G. Knizia, H.-J. Werner, J. Chem. Phys. 2007, 127, 221106-1-4; b) G. Knizia,T. B. Adler, H.-J. Werner, J. Chem. Phys. 2009, 130, 054104-1-20.

[21] G. Jansen, B. A. Heß, Phys. Rev. A 1989, 39, 6016-6017.

[22] CCSD(T)-F12a- und F12-b-Berechnungen wurden mit einem Gaussorbital-Basissatz augmentierter triple-zeta-valence-Qualität durchgeführt (aug-cc-pVTZ; siehe: R. A. Kendall, T. H. Dunning, Jr., R. J. Harrison, J. Chem. Phys. 1992, 96, 6796-6806.), wobei die passenden Hilfsbasissätze für die verwendeten Näherungen (density-fitting (DF) und Einheitseinschub (RI)) zum Einsatz kamen (a) F. Weigend, Phys. Chem. Chem. Phys. 2002, 4, 4285-4291; b) F. Weigend, A. Köhn, C. Hättig, $J$. Chem. Phys. 2002, 116, 3175-3183). Die Rechnungen wurden mit dem Programpaket Molpro ausgeführt (MOLPRO, Version 2010.1, ein Paket von ab-initio-Programmen, H.-J. Werner, P. J. Knowles, G. Knizia, F. R. Manby, M. Schütz, P. Celani, T. Korona, R. Lindh, A. Mitrushenkov, G. Rauhut, K. R. Shamasundar, T. B. Adler, R. D. Amos, A. Bernhardsson, A. Berning, D. L. Cooper, M. J. O. Deegan, A. J. Dobbyn, F. Eckert, E. Goll, C. Hampel, A. Hesselmann, G. Hetzer, T. Hrenar, G. Jansen, C. Köppl, Y. Liu, A. W. Lloyd, R. A. Mata, A. J. May, S. J. McNicholas, W. Meyer, M. E. Mura, A. Nicklass, D. P. O'Neill, P. Palmieri, K. Pflüger, R. Pitzer, M. Reiher, T. Shiozaki, H. Stoll, A. J. Stone, R. Tarroni, T. Thorsteinsson, M. Wang, A. Wolf, siehe http://www.molpro.net.). Die kombinierten Effekte von relativistischen Korrekturen und Core-Valenzelektronenkorrelation des Bromatoms wurden durch Standard-CCSD(T)-Berechnungen mit dem relativistischen Douglas-Kroll-Heß-Hamiltonoperator zweiter Ordnung ermittelt, wobei der Basissatz für Brom durch aug-cc-pwCVTZ-DK (B. Prascher, D. E. Woon, K. A. Peterson, T. H. Dunning, Jr., A. K. Wilson, Theor. Chem. Acc. 2011, 128, 69-82.) und der für Stickstoff durch aug-ccpVTZ-DK ersetzt und die 3d-Schale des Bromatoms in die Korrelationsbehandlung einbezogen wurde. Die Veränderung der betrachteten molekularen Eigenschaft gegenüber dem nicht-relativistischen CCSD(T)/aug-cc-pVTZResultat ohne 3d-Korrelation wurde dann zum entsprechenden CCSD(T)F12a-Wert addiert. Weitere Details und (nahezu identische) CCSD(T)-F12bResultate finden sich im Supplement.

[23] K. P. Huber, G. Herzberg, "Constants of Diatomic Molecules" (data prepared by J. W. Gallagher and R. D. Johnson, III) in NIST Chemistry WebBook, NIST Standard Reference Database Number 69, Eds. P. J. Linstrom, W. G. Mallard, National Institute of Standards and Technology, Gaithersburg MD, 20899, http://webbook.nist.gov.

[24] Mit dem Begriff "Kontakt" werden im Folgenden Abstände unterhalb der Summe der van-der-Waals Radien beschrieben.

[25] Der Basissatzsuperpositionsfehler wurde durch Anwendung der Counterpoise-Korrektur berücksichtigt (S. F. Boys, F. Bernardi, Mol. Phys. 1970, 19, 553-566.).

[26] Siehe folgende und darin zitierte Literatur: A. D. Buckingham, J. E. Del Bene, S. A. C. McDowell, Chem. Phys. Lett. 2008, 463, 1-10. [27] a) G. Jansen, A. Heßelmann, J. Phys. Chem. A 2001, 105, 1115611157; b) A. Heßelmann, G. Jansen, Phys. Chem. Chem. Phys. 2003, 5, 5010-5014; c) A. J. Misquitta, R. Podeszwa, B. Jeziorski, K. Szalewicz, J. Chem. Phys. 2005, 123, 214103-1-14.

[28] DFT-SAPT-Berechnungen wurden mit der density-fitting-Näherung wie im Molpro implementiert (A. Heßelmann, G. Jansen, M. Schütz, $J$. Chem. Phys. 2005, 122, 014103-1-17.) unter Verwendung von aug-ccpVTZ- und aug-cc-pVQZ-Orbitalbasissätzen und den oben angegebenen passenden Hilfsbasissätzen durchgeführt. Dispersions- und AustauschDispersionsenergien wurden mit Orbitalen berechnet, die mit einer asymptotisch korrigierten Variante des Perdew-Burke-Ernzerhof AustauschKorrelationspotentials (PBEAC) bestimmt wurden, in Kombination mit der adiabatischen lokalen Dichtenäherung (ALDA) für den AustauschKorrelationskernel. Die verbleibenden DFT-SAPT-Beiträge sowie die Partialladungsanalyse wurden mit dem entsprechenden Hybrid-AustauschKorrelationspotential, welches $25 \%$ exakten Austausch beinhaltet, erhalten (PBE0AC, siehe A. Heßelmann, G. Jansen, Chem. Phys. Lett. 2002, 357, 464-470; A. Heßelmann, G. Jansen, Chem. Phys. Lett. 2002, 362, 319-325.). Die Dispersions- und Austausch-Dispersionsbeiträge zweiter Ordnung 
wurden im Beitrag $\mathrm{E}_{\text {disp }}$ zusammengefasst, welcher zu seinem Grenzwert für den vollständigen Basissatz mit Hilfe der $1 / \mathrm{X}^{3}$-Zweipunktstandardformel für $X=3$ und 4 extrapoliert wurde (A. Halkier, T. Helgaker, P. Jørgensen, W. Klopper, H. Koch, J. Olsen, A. K. Wilson, Chem. Phys. Lett. 1998, 286, $243-$ 252). Die verbleibenden Beiträge wurden direkt den aug-cc-pVQZ-

Resultaten entnommen. Während $\mathrm{E}_{\mathrm{el}}$ und $\mathrm{E}_{\text {exch }}$ die elektrostatischen und

Austauschbeiträge erster Ordnung bedeuten, steht $\mathrm{E}_{\text {ind }}$ für die Summe aus

Induktions- und Austausch-Induktionsbeiträgen zweiter Ordnung sowie der

$\delta(\mathrm{HF})$-Abschätzung der Beiträge höherer Ordnung.
[29] a) A. E. Reed, R. B. Weinstock, F. Weinhold, J. Chem. Phys. 1985, 83, 735-746; b) A. E. Reed, L. A. Curtis, F. Weinhold, Chem. Rev. 1988, 88, 899-926.

[30] Der mesomeren Struktur mit entgegengesetzten Formalladungen an $\mathrm{N}_{\beta}$ und $\mathrm{N}_{\gamma}$ wurde von Klapötke et al. eine bedeutende Rolle bei der Festkörperstruktur von $\mathrm{HN}_{3}$ zugesprochen. Für die Struktur von $\mathrm{BrN}_{3}$ spielt diese Grenzform nach unseren Rechnungen dagegen keine Rolle. 


\section{Entry for the Table of Contents}

Layout 1:

\section{Bromine Azide}

Benjamin Lyhs, Dieter Bläser, Christoph Wölper, Stephan Schulz*, Georg Jansen Page - Page

Festkörperstruktur von Bromazid

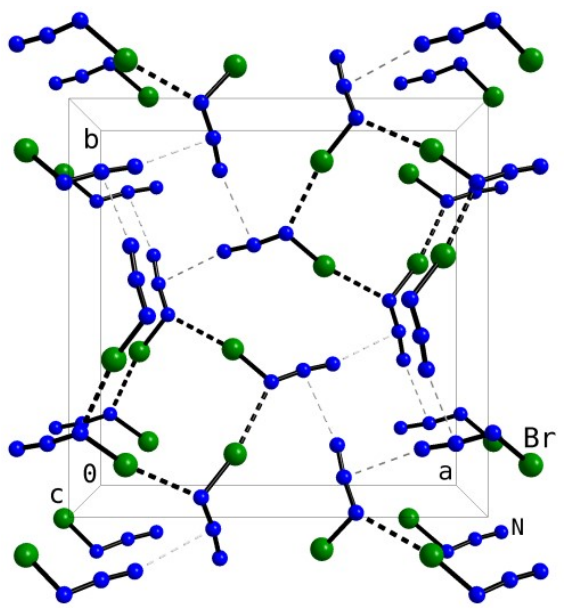

Helicale Struktur von $\mathrm{BrN}_{3}$ im

Festkörper - ein neuartiges

Strukturelement für kovalente p-BlockAzide. 


\section{DuEPublico}

Duisburg-Essen Publications online

\section{DEU ${ }^{\prime} I_{S} S_{N} B_{N} U$ R G}

offen im Denken

ub

universitäts bibliothek

This text is made available via DuEPublico, the institutional repository of the University of Duisburg-Essen. This version may eventually differ from another version distributed by a commercial publisher.

DOI: $\quad 10.1002 /$ ange.201108092

URN: urn:nbn:de:hbz:464-20201112-094956-3

This is the peer reviewed version of the following article: Angew. Chem. 2012,124, 2008-2013, which has been published in final form at: https://doi.org/10.1002/ange.201108092

All rights reserved. 